\title{
AN EMBEDDING CONSTRUCTION FOR ORDERED GROUPS
}

\author{
VAHAGN H. MIKAELIAN
}

\section{To Professor Alexander Yurievich Ol'shanskii, my teacher}

(Received 20 January 2000; revised 3 March 2002)

Communicated by R. B. Howlett

\begin{abstract}
Generalizing and strengthening some well-known results of Higman, B. Neumann, Hanna Neumann and Dark on embeddings into two-generator groups, we introduce a construction of subnormal verbal embedding of an arbitrary (soluble, fully ordered or torsion free) ordered countable group into a twogenerator ordered group with these properties. Further, we establish subnormal verbal embedding of defect two of an arbitrary (soluble, fully ordered or torsion free) ordered group $G$ into a group with these properties and of the same cardinality as $G$, and show in connection with a problem of Heineken that the defect of such an embedding cannot be made smaller, that is, such verbal embeddings of ordered groups cannot in general be normal.
\end{abstract}

2000 Mathematics subject classification: primary 20E10, 20E $15,20 \mathrm{E} 22,20 \mathrm{~F} 14,06 \mathrm{~F} 15$.

Keywords and phrases: embeddings of groups, ordered groups, two-generator groups.

\section{Introduction}

The famous theorem of Higman, B. Neumann and Hanna Neumann on embedding of an arbitrary countable group into a two-generator group [7] was an initial step for further research on embeddings in two-generator groups with additional properties. Let us list some of the main results in this direction, connected to the subject of the current paper.

Embeddings of soluble groups Since abelian (or nilpotent) groups are not in general embeddable into two-generator abelian (or nilpotent) groups [21, 22], such embeddings of soluble groups are of special interest. B. Neumann and Hanna Neumann have 
proved that every soluble countable group of length $l$ is embeddable into a soluble two-generator group of length at most $l+2$ (but not $l+1$ in general) [22].

Subnormal embeddings Dark has shown in [1] that every countable group can be embedded in a two-generator group in such a way that its image is a subnormal subgroup of the two-generator group. (In this connection see also the paper of Hall [4].)

Embeddings into a word subgroups B. Neumann and Hanna Neumann have constructed in [22] not simply an embedding into a two-generator group but also into the second derived group of the latter (see also [1]). Since two-generator groups also are countable we can, for arbitrary $n$, construct embeddings of this type into the $n$th member of the derived series of a suitable two-generator group. But one can set the problem in a much more general form: whether for a given non-trivial word set $V \subseteq F_{\infty}$ it is possible to embed a given countable group into the verbal subgroup (corresponding to $V$ ) of some two-generator group.

Embeddings of ordered groups Solving a problem offered by himself, B. Neumann has proved that it is possible to embed a given countable (soluble) fully ordered group into a two-generator (soluble) fully ordered group [20] (where of course it is assumed that the order of the two-generator group 'continues' the order of the isomorphic copy of the initial group).

For other results in this direction we refer to the papers of Ol'shanskii $[25,26]$, B. Neumann [21], Levin and Rosenberger [13], Hall [4], Wilson and Zalesskii [28] and to literature cited there.

The main result of this paper (Theorem 1 in Section 2) generalizes or strengthens the theorems listed above, for it establishes an embedding combining all the properties we have mentioned. For an arbitrary non-trivial word set $V$, an arbitrary countable group $G$ is subnormally embeddable into a two-generator group $G_{2}$, its image lying in $V\left(G_{2}\right)$, and if $G$ is soluble, fully ordered or torsion free, the group $G_{2}$ 'inherits' these properties. The proof of this result occupies Section 2 and presents the main construction of the paper.

In Section 3 we deal with soluble embeddings of ordered groups and normal embeddings of ordered groups. First we see that the value $l+2$ for the soluble length of the two-generator group obtained in [22] cannot be combined with 'verbality' of the embedding of Theorem 1. On the other hand we can construct an embedding preserving the rest of the properties listed above: every ordered soluble countable group $G$ of soluble length $l$ can be subnormally embedded into an ordered twogenerator soluble group of length $l+2$, but not $l+1$ in general (Theorem 2 ). This theorem strengthens Theorem 3.3 and Corollary 3.4 of [20] as well as Theorem 5.1 and Corollary 5.2 of [22] by subnormality, and Theorem 2 of [1] by full order and by solubility. 
A simplified version of our construction gives a subnormal embedding of defect 2 of an arbitrary (ordered, soluble or torsion free) group $G$ into an (ordered, soluble or torsion free) group $G_{1}$ of the same cardinality as $G$, such that the image of $G$ lies in $V\left(G_{1}\right)$ for a given non-trivial word set $V$ (Theorem 3 ). This strengthens the statement $A$ of our main theorem in [15] and is connected with the problem of Heineken on normal verbal embeddability [5]: whether for a given non-trivial word set $V$ and for a given group $G$ there exists a group $H$ with a normal subgroup $\tilde{G}$ such that $\tilde{G}$ lies in $V(H)$ and is isomorphic to $G$. (See the criterion of verbal normal embeddings in our recent paper [6]). We will see here that the defect 2 obtained above cannot be made smaller: there exists a fully ordered (nilpotent, torsion free) group $G$ that, for a certain word set $V$, cannot be normally embedded into a group $H$ so that the image of $G$ lies in $V(H)$ (even without the requirement on $H$ to be ordered, soluble, torsion free, or of the same cardinality as $G$ ).

And finally, we would like to announce here that the method we use in the current paper can be modified for the case of generalized soluble groups. Kovács and B. Neumann have extended in [10] the result of [22] and have constructed embeddings of countable $S I^{*}$-groups (of countable $S N^{*}$-groups) into two-generator $S I^{*}$-groups (into two-generator $S N^{*}$-groups, respectively). It is very natural to ask whether one can 'add full order' to this embedding as well. Recently we have constructed such subnormal embeddings for fully ordered and generalized soluble groups ( $S N-, S I-, S N^{*}$ or $S I^{*}$-groups) into appropriate fully ordered and generalized soluble two-generator groups [17].

Notations For information on varieties of groups we refer to the book of Hanna Neumann [23]. Absolutely free groups of infinite rank (or of finite rank $n$ ) will be denoted by $F_{\infty}$ (or by $F_{n}$ ). For the given group $G$, we denote, as usual, by $\operatorname{var}(G)$ the variety generated by $G$. We have $\operatorname{var}(G)=\operatorname{var}\left(F_{\infty} / V\left(F_{\infty}\right)\right)$, where $V$ is the set of all identities satisfied in $G$. We reserve the German letters $\mathfrak{A}, \mathfrak{N}_{c}$ and $\mathfrak{O}$ for the varieties of all abelian groups, all nilpotent groups of class at most $c$, and all groups respectively.

For information on wreath products we refer to the paper of P. Neumann [24] or to the book of Kargapolov and Merzljakov [9]. Since we use wreath products repeatedly, let us reserve Greek letters to denote elements of the base group, and Roman letters for elements of the 'active group'. The support of the element $\varphi$ of the base group of the (complete) wreath product $A \mathrm{Wr} B$ is denoted by $\sigma(\varphi)$.

For information on ordered groups we refer to the papers of B. Neumann [19], Levi $[11,12]$, or to the book of Fuchs [2]. The group $G$ is fully ordered if there is a transitive binary relation $<$ defined on $G$ such that for each $a, b \in G$ one and only one of the three alternatives $a<b, a=b$ and $b<a$ holds, and if $a<b$ then $a c<b c$ and $c a<c b$ hold for all $c \in G$. These groups are often called O-groups, but we adopt 
the term used by B. Neumann in [20]. The groups $A$ and $B$ are order isomorphic if there exists an order-preserving isomorphism $f: A \rightarrow B$. For an ordered group $G$ we denote by $G^{+}$and $G^{-}$the sets of 'positive' and 'negative' elements: an element $x$ is positive if $1<x$, and $x$ is negative if $x<1$.

Let us make the following two conventions. Firstly, if we have an isomorphic embedding $\beta: G \rightarrow H$, we avoid the details immaterial to our purposes and use in proofs the same notation for the group $G$ and its image in $H$. Secondly, if in this situation the group $G$ is an ordered one and if we have defined an order relation on $H$ such that its reduction on $\beta(G)$ makes the latter order isomorphic to $G$, we will use the same sign ' $<$ ' for the orders on $G$ and on $H$.

\section{Construction of the main embedding}

THEOREM 1. Let $G$ be an arbitrary countable group and $V \subseteq F_{\infty}$ be an arbitrary non-trivial word set. Then there exists a two-generator group $G_{2}$ with a subgroup $\tilde{G}$ such that:

(1) $G$ is isomorphic to $\tilde{G}$.

(2) $\tilde{G}$ is subnormal in $G_{2}$, and lies in the verbal subgroup $V(G)$.

Moreover if the group $G$ satisfies some of the following conditions

(a) $G$ is soluble,

(b) $G$ is a fully ordered group,

(c) $G$ is torsion free,

then the two-generator group $G_{2}$ can be chosen to satisfy the same conditions.

As we will see from the proof, the group $G_{2}$ can be chosen to belong to variety $\operatorname{var}(G) \mathfrak{N}_{c} \mathfrak{A}^{2}$, where as $c$ we can take the nilpotency class of an arbitrary group not from the variety corresponding to the word set $V$.

Here we will pay most attention to subnormal, verbal embeddings of fully ordered groups; the condition (a) will be obtained as a property of the structure of the proof. In the case when $G_{2}$ is fully ordered the condition (c) is guaranteed automatically [19]. The case when $G$ is not necessarily an ordered group is covered by statements $\mathbf{B}$ and C of our Theorem 1 in [15] (announced at the ICM 1998, Berlin [14]). In this case the fact that the two-generator group is torsion free follows from the proof in [15, Section 2].

Let us note that we cannot replace the property (c) by the property that $G_{2}$ is a periodic group because such a property cannot be combined with (b) if the group $G$ is fully ordered, and cannot be combined with (a) in the general case.

We begin with a useful criterion for fully ordered groups adopted from the paper of Levi [11]. 
LEMMA 1 (Levi). The group $G$ is fully ordered if and only if it can be presented as a union $G=G^{-} \cup\{1\} \cup G^{+}$such that $G^{-}$and $G^{+}$are semigroups, and $g^{-1} G^{+} g \subseteq G^{+}$ for all $g \in G$.

If the given group is represented in the form $G=G^{-} \cup\{1\} \cup G^{+}$and the conditions of Lemma 1 hold, one can set for $a, b \in G$

$$
a<b \text { if and only if } a^{-1} b \in G^{+} .
$$

Next we construct a nilpotent group $S$ essential for our proof. We have to note that the proof of the following lemma could be somewhat shorter if we simply use [19, Theorem 2.3] to show that $S$ is fully ordered. But we define the order on this group explicitly in order to have an explicit order on $G_{2}$.

LEMMA 2. For arbitrary non-trivial word set $V$ there exists a fully ordered nilpotent torsion free group $S$ with a non-trivial element a such that $a \in V(S)$ and $1<a^{i}$ for all positive integers $i$.

PROOF. The set of all finite nilpotent groups generates the variety of all groups $\mathfrak{O}$ [23]. Since $V$ is non-trivial there exists a nilpotent group not from the variety $\operatorname{var}\left(F_{\infty} / V\left(F_{\infty}\right)\right)$ corresponding to $V$. Thus there is a free nilpotent group $S=F_{n}\left(\mathfrak{N}_{c}\right)$ of some rank $n$ and of some class $c$ which does not belong to $\operatorname{var}\left(F_{\infty} / V\left(F_{\infty}\right)\right)$. Then $V(S) \neq\{1\}$ and $V(S)$ contains an element $a$ of infinite order. Let further $S_{i}=\gamma_{i}(S)$ be the $i$ th member of the lower central series of $S$ :

$$
S=S_{1} \geq S_{2} \geq \cdots \geq S_{c+1}=\{1\} .
$$

First we define (full) orders on factors $S_{i} / S_{i+1}$. Each of these factors is a free abelian (torsion free) group of finite rank. A direct product of finitely many infinite cycles can be fully ordered in the following way [12]: order each cycle $Z=\langle z\rangle$ as follows:

$$
z^{i}<z^{j} \quad \text { if and only if } i<j
$$

and continue these orders on the direct product lexicographically: compare elements $\bar{z}_{1}, \bar{z}_{2} \in S_{i} / S_{i+1}$ and set $\bar{z}_{1}<\bar{z}_{2}$ if the first coordinate of $\bar{z}_{1}$ is less than that of $\bar{z}_{2}$; if their first coordinates are equal we consider the second ones, etc ...

Now we continue orders already defined on the factors on the whole group $S$. Denote by $S_{c}^{+}$and $S_{c}^{-}$the semigroups of positive and negative elements of $S_{c}$ and continue by induction:

$$
\begin{aligned}
& S_{c-i}^{+}=\left\{x \in S_{c-i} \mid 1<x \quad\left(\bmod S_{c-i+1}\right)\right\}, \\
& S_{c-i}^{-}=\left\{x \in S_{c-i} \mid x<1 \quad\left(\bmod S_{c-i+1}\right)\right\}
\end{aligned}
$$


Consider the sets $S^{+}=\bigcup_{i=0}^{c-1} S_{c-i}^{+}$and $S^{-}=\bigcup_{i=0}^{c-1} S_{c-i}^{-}$. These satisfy the conditions of Lemma 1; the verification is easy and can by found in [19] for more general situations. Therefore the full order we are looking for can be defined as:

$$
\text { for } x, y \in S, x<y \text { if and only if } x^{-1} y \in S^{+} .
$$

Now return to the element $a$. Since $a \neq 1$ we have $1<a$ or $a<1$. We can assume $1<a$ because we can always replace our order ' $<$ ' by the inverse order relation ' $<^{-1}$ '. And finally since $a$ is positive its powers $a^{2}, a^{3}, \ldots$, also are positive according to definition of full order.

For the given (not necessarily ordered or countable) group $G$ let us consider the (complete) wreath product $G \mathrm{Wr} S$ with the base group $G^{S}$. Choose a subset $\Omega$ of elements $\chi_{8}$ of $G^{S}$ in the following way

$$
\chi_{g}(s)= \begin{cases}g, & \text { if } s=a^{i}, i=0,1,2, \ldots ; \\ 1, & \text { if } s \in S \backslash\left\{a^{i} \mid i=0,1,2, \ldots\right\} .\end{cases}
$$

(Here $a$ is the element defined in Lemma 2.)

Define a subgroup of $G \mathrm{Wr} S$ :

$$
G_{1}=\langle\Omega, S\rangle
$$

LEMMA 3. If $G, V$ and $G_{1}$ are defined as above, the group $G_{1}$ is of the same cardinality as $G ; G$ can be subnormally embedded into $G_{1}$ such that its image lies in $V\left(G_{1}\right)$; and if $G$ is a fully ordered group, $G_{1}$ can be fully ordered in such a way that $G$ is order isomorphic to its image in $G_{1}$ (relative to the reduction of the order of $G_{1}$ ).

PROOF. $V\left(G_{1}\right)$ is non-trivial since $V(S)$ is non-trivial: $V\left(G_{1}\right)$ contains the element $a$ (and all its powers). Define $G_{0}$ to be the first copy of $G$ in the base group $G^{s}$ :

$$
\varphi_{g} \in G_{0} \quad \text { if and only if } \begin{cases}\varphi_{g}(s)=g, & \text { if } s=1 ; \\ \varphi_{g}(s)=1, & \text { if } s \in S \backslash\{1\} .\end{cases}
$$

Since a verbal subgroup is normal we have

$$
a^{\chi_{g}}=\chi_{g}^{-1} a \chi_{g}=a\left(\chi_{g}^{-1}\right)^{a} \chi_{g} \in V\left(G_{1}\right) .
$$

But as straightforward computations show,

$$
\left(\left(\chi_{g}^{-1}\right)^{a} \chi_{g}\right)(s)= \begin{cases}g, & \text { if } s=1=a^{0} \\ 1, & \text { if } s=a, a^{2}, a^{3}, \ldots \\ 1, & \text { if } s \in S \backslash\left\{a^{i} \mid i=0,1,2, \ldots\right\}\end{cases}
$$


Thus $\left(\chi_{g}^{-1}\right)^{a} \chi_{g}=\varphi_{g} \in G_{0}$, and the first copy of $G$ lies in $V\left(G_{1}\right)$ for $\varphi_{g}=a^{-1} a^{\chi_{s}} \in$ $V\left(G_{1}\right)$. The rule $g \mapsto \varphi_{g}$ defines an embedding of $G$ into $G \mathrm{Wr} S$ which we denote by $\nu$.

Since $G_{1}$ is generated by $\Omega$ and $S$, card $\left(G_{1}\right)=$ card $(\Omega)$ because $S$ is countable. (The case of finite $G$, when the cardinality of a group need not necessarily coincide with the cardinality of its generating system is impossible here since the orered group $G$ is torsion free.) On the other hand, clearly card $(\Omega)=$ card $(G)$.

Further we note that for each element $s \chi \in G_{1}$ the support $\sigma(\chi)$ is well-ordered (according to the order defined on $S$ in Lemma 2). This follows from the fact that the supports of all elements of type $\chi_{g}$ are well-ordered and from the following obvious properties of elements of the base group of a wreath product: if $\psi, \psi_{1}, \psi_{2} \in G^{S}$ and $s \in S$ then

$$
\begin{aligned}
\sigma\left(\psi^{-1}\right) & =\sigma(\psi), \\
\sigma\left(\psi_{1} \cdot \psi_{2}\right) & \subseteq \sigma\left(\psi_{1}\right) \cup \sigma\left(\psi_{1}\right), \\
\sigma\left(\psi^{s}\right)=\sigma(\psi) \cdot s & =\left\{s^{\prime} \cdot s \mid s^{\prime} \in \sigma(\psi)\right\} .
\end{aligned}
$$

Now we are able to continue the full order of $S$ to a full order on the whole group $G_{1}$. For two non-equal elements $s_{1} \chi_{1}, s_{2} \chi_{2} \in G_{1}$ define

$$
s_{1} \chi_{1}<s_{2} \chi_{2}
$$

if and only if $s_{1}<s_{2}$ or $s_{1}=s_{2}$ and $\chi_{1}\left(s_{0}\right)<\chi_{2}\left(s_{0}\right)$, where $s_{0}$ is the 'first coordinate' (the least element of $S$ according to the order defined on the group $S$ ) such that $\chi_{1}\left(s_{0}\right) \neq \chi_{2}\left(s_{0}\right)$. (Clearly $\chi_{1}(s)=\chi_{2}(s)$ holds for infinitely many 'first' values of $s$.) Existence of such a coordinate $s_{0}$ follows from the fact that $\sigma\left(\chi_{1}\right)$ and $\sigma\left(\chi_{2}\right)$ are well-ordered and $s_{1} \chi_{1} \neq s_{2} \chi_{2}$.

This order relation is a full order. Firstly it is easy to see that this relation is transitive and that for arbitrary elements $s_{1} \chi_{1}, s_{2} \chi_{2} \in G_{1}$ one and only one of following three alternatives can take place

$$
s_{1} \chi_{1}<s_{2} \chi_{2}, \quad s_{1} \chi_{1}=s_{2} \chi_{2}, \quad s_{2} \chi_{2}<s_{1} \chi_{1} .
$$

Assume further $s_{1} \chi_{1}<s_{2} \chi_{2}$ and $s \chi \in G_{1}$. It remains to show that

$$
\begin{aligned}
& s_{1} \chi_{1} \cdot s \chi=s_{1} s\left(\chi_{1}\right)^{s} \chi<s_{2} s\left(\chi_{2}\right)^{s} \chi=s_{2} \chi_{2} \cdot s \chi, \\
& s \chi \cdot s_{1} \chi_{1}=s s_{1}(\chi)^{s_{1}} \chi_{1}<s s_{2}(\chi)^{s_{2}} \chi_{2}=s \chi \cdot s_{2} \chi_{2} .
\end{aligned}
$$

Let us prove the first of these relations. It is obvious that if $s_{1}<s_{2}$ then $s_{1} s<s_{2} s$, for ' $<$ ' is a full order on $S$. Assume $s_{1}=s_{2}$. Since $\sigma\left(\left(\chi_{1}\right)^{s}\right)=\sigma\left(\chi_{1}\right) \cdot s$ and $\sigma\left(\left(\chi_{2}\right)^{s}\right)=\sigma\left(\chi_{2}\right) \cdot s$ we get

$$
\left(\chi_{1}\right)^{s}<\left(\chi_{2}\right)^{s} \text { if and only if } \chi_{1}<\chi_{2} .
$$


And clearly

$$
\left(\chi_{1}\right)^{s}<\left(\chi_{2}\right)^{s} \text { if and only if }\left(\chi_{1}\right)^{s} \cdot \chi<\left(\chi_{2}\right)^{s} \cdot \chi .
$$

because the simultaneous multiplication of some of coordinates of $\left(\chi_{1}\right)^{s}$ and of $\left(\chi_{2}\right)^{s}$ by the same elements (coordinates of $\chi$ ) does not change the relation $\left(\chi_{1}\right)^{s}<\left(\chi_{2}\right)^{s}$.

It remains to note that the reduction of this order of the group $G_{1}$ on the first copy $G_{0}$ makes the latter order isomorphic to $G$.

As the next step we must embed $G_{1}$ subnormally into a group $D$ such that

(1) $D$ is of the same cardinality as $G_{1}$.

(2) The isomorphic image of $G_{1}$ under this embedding lies in the derived subgroup $D^{\prime}$.

(3) The group $D$ can be fully ordered in such a way that the reduction of the order on the isomorphic image of $G_{1}$ makes this image order isomorphic to $G_{1}$.

Of course we could get such a group $D$ simply from Lemma 3 and Lemma 2 taking the commutator word $\left[x_{1}, x_{2}\right]=x_{1}^{-1} x_{2}^{-1} x_{1} x_{2}$ instead of general word set $V$. As $S$ we could then use the $\mathfrak{N}_{2}$-free group $F_{2}\left(\mathfrak{N}_{2}\right)$. Then the group $D$ would belong to variety $\operatorname{var}\left(G_{1}\right) \cdot \mathfrak{N}_{2}$. Nevertheless we will take another construction (given by P. Neumann [24] and frequently used in literature) not only because it allows a more 'economical' embedding (namely into a group in the variety $\operatorname{var}\left(G_{1}\right) \mathfrak{A}$ ) but also because the same construction will be used in the next section.

LEMMA 4. For an arbitrary fully ordered group $G_{1}$ there exists a fully ordered group $D$ which belongs to variety $\operatorname{var}\left(G_{1}\right) \mathfrak{A}$ and which satisfies the conditions (1), (2) and (3) listed above.

Proof. Let $C=\langle c\rangle$ be an infinite cycle generated by the element $c$. The group $G_{1}$ is subnormally embeddable into the wreath product $G_{1} \mathrm{Wr} C$, mapping onto its first copy in the base group $G_{1}^{C}$. Let $\psi_{g}$ be the element of this first copy corresponding to the element $g \in G_{1}$.

We choose $D$ to be the subgroup of this wreath product generated by $c$ and by the following elements $\pi_{g}$ in $G_{1}^{C}\left(g \in G_{1}\right)$ :

$$
\pi_{g}\left(c^{i}\right)= \begin{cases}g, & \text { if } i \geq 0 \\ 1, & \text { if } i<0\end{cases}
$$

For arbitrary $g \in G_{1}$,

$$
\left[\pi_{g^{-1}}, c\right]\left(c^{i}\right)=\left(\pi_{g^{-1}}^{-1} \pi_{g^{-1}}^{c}\right)\left(c^{i}\right)=\left(\pi_{g} \pi_{g^{-1}}^{c}\right)\left(c^{i}\right)= \begin{cases}g, & \text { if } i=0 \\ 1, & \text { if } i \neq 0\end{cases}
$$


Thus $\left[\pi_{g^{-1}}, c\right]=\psi_{g}$ and therefore the first copy of $G_{1}$ lies in the derived subgroup $D^{\prime}$. Let us denote by $\lambda$ the embedding $g \mapsto \psi_{g}$ of $G_{1}$ onto the first copy of $G_{1}$ in $G_{1}^{C}$.

For each element $c^{i} \pi \in D$ the support $\sigma(\pi)$ has a minimal element. Let us define the following order relation on $D: c^{i} \pi_{1}<c^{j} \pi_{2}$ if and only if $i<j$ or if $i=j$ and $\pi_{1}\left(c^{k}\right)<\pi_{2}\left(c^{k}\right)$, where $c^{k}$ is the least power of $c$ for which $\pi_{1}\left(c^{k}\right)$ and $\pi_{2}\left(c^{k}\right)$ are different. In analogy with the proof of Lemma 3 we see that we have defined a full order on $D$ and that its reduction on the first copy of $G_{1}$ makes that copy order isomorphic to $G_{1}$. Clearly $D$ belongs to variety $\operatorname{var}\left(G_{1}\right) \mathfrak{A}$ and is of the same cardinality as $G_{1}$.

Now assume our initial group $G$ to be countable. Then the corresponding group $D$ is also countable. We take another infinite cycle $Z=\langle z\rangle$ and embed $D$ into the wreath product $D \mathrm{Wr} Z$, again onto the first copy of $D$ in the base group $D^{Z}$. We can order the elements of $D$ linearly, $D=\left\{d_{0}, d_{1}, \ldots, d_{n}, \ldots\right\}$, and define a special element $\omega$ of $D^{Z}$ :

$$
\omega\left(z^{i}\right)= \begin{cases}d_{k}, & \text { if } i=2^{k}, k=0,1,2, \ldots ; \\ 1, & \text { if } i \in \mathbb{Z} \backslash\left\{2^{k} \mid k=0,1,2, \ldots\right\} .\end{cases}
$$

We use an idea of Hall from [3]. For arbitrary $d_{n}$ we have $\omega^{\left(z^{-2^{n}}\right)}(1)=d_{n}$. So for arbitrary $d_{n}$ and $d_{m}$ :

$$
\left[\omega^{\left(z^{-2^{n}}\right)}, \omega^{\left(z^{-2^{m}}\right)}\right](1)=\left[d_{n}, d_{m}\right]
$$

We note further that for arbitrary $j \neq 0$

$$
\left[\omega^{\left(z^{-2^{n}}\right)}, \omega^{\left(z^{-2^{m}}\right)}\right]\left(z^{j}\right)=1,
$$

because for $u_{1}, v_{1}, u_{2}, v_{2} \in \mathbb{Z}$ and $u_{1} \neq u_{2}$ the equation $2^{u_{1}}-2^{v_{1}}=2^{u_{2}}-2^{v_{2}}$ has only one solution: $u_{1}=v_{1}$ and $u_{2}=v_{2}$.

Thus every element of the derived group $D^{\prime}$ belongs to the derived group of the two-generator group $G_{2}=\left\langle\omega, z^{-1}\right\rangle$. The group $G_{1}$ is embeddable into two-generator group $G_{2}$, for it is embeddable into $D^{\prime}$. Moreover, the following holds:

LEMMA 5. If $G_{1}$ and $G_{2}$ are the groups constructed above, then $G_{1}$ can be subnormally embedded in $G_{2}$, and $G_{2}$ can be ordered in such a way that $G_{1}$ is order isomorphic to its image in $G_{2}$.

Proof. $G_{1}$ is subnormal in $D^{\prime}$ because $G_{1}$ is subnormal in $D$. The derived group $D^{\prime}$ is subnormal in $G_{2}$ because it is subnormal in $D \mathrm{Wr} Z$. ( $D^{\prime}$ is normal in the base group $D^{Z}$, for $D^{\prime}$ is characteristic in the first copy and the latter is normal in the base group.) 
Let us consider the subgroup $N \leq D \mathrm{Wr} Z$ generated by the first copy of $D$ and the elements $\omega$ and $z$. We note that for each element $z^{i} \delta \in N$ the support $\sigma(\delta)$ has a minimal element. Thus we define an order similar to that of Lennma 4: $z^{i} \delta_{1}<z^{j} \delta_{2}$ if and only if $i<j$ or if $i=j$ and $\delta_{1}\left(z^{k}\right)<\delta_{2}\left(z^{k}\right)$, where $z^{k}$ is the least power of $z$ such that $\delta_{1}\left(z^{k}\right)$ is different from $\delta_{2}\left(z^{k}\right)$. We have defined a full order on $N$ and its reduction on the first copy of $D$ makes that copy order isomorphic to $D$. Clearly $N$ belongs to the variety $\operatorname{var}(D) \mathfrak{A}$. Now simply choose the order on the group $G_{2}$ to be the reduction of the order we have just defined on $N$ to its subgroup $G_{2}$.

Denote by $\theta$ the subnormal embedding of $D^{\prime}$ in $G_{2}$ obtained in Lemma 5. Now we are able to present a subnormal verbal embedding $\mu$ of $G$ into the two-generator group $G_{2}$ as follows:

$$
\mu=\nu \lambda \theta,
$$

where $G \stackrel{\nu}{\triangleleft} \triangleleft G_{1} \stackrel{\lambda}{\triangleleft \triangleleft} D^{\prime} \stackrel{\theta}{\triangleleft} G_{2}$. The defect of $G$ in $G_{2}$ is 6 , for the defect of each of these embeddings is 2 . The group $G_{2}$ belongs to the variety var $(G) \mathfrak{N}_{c} \mathfrak{A}^{2}$.

To conclude the proof of Theorem 1 it remains to notice that if the group $G$ is soluble, say of length $l$, the group $G_{2}$ is also soluble, having length $l+c+2$, and if the group $G$ is torsion free, the two-generator group $G_{2}$ is also torsion free, for it is a subgroup of the torsion free group ( $(G \mathrm{Wr} S) \mathrm{Wr} C) \mathrm{Wr} Z)$. Theorem 1 is proved.

\section{Some related problems}

As we mentioned in the introduction, the result of B. Neumann and Hanna Neumann given in [22] guarantees not only embeddability of a soluble countable group of length $l$ into a soluble two-generator group, but also that the length of the latter is at most $l+2$. This is the best possible general value because according to [22, Lemma 5.3] there is an abelian group which cannot be embedded in a two-generator metabelian group. (See also [8].)

It is easy to see that this condition on the solubility length cannot be strengthened by requirement of 'verbality' of the embedding. For if $V$ contains the commutator word $\delta_{t}\left(x_{1}, \ldots, x_{2^{\prime}}\right)$,

$$
\delta_{0}=x, \quad \delta_{t}\left(x_{1}, \ldots, x_{2^{\prime}}\right)=\left[\delta_{t-1}\left(x_{1}, \ldots, x_{2^{\prime-1}}\right), \delta_{t-1}\left(x_{2^{t-1}+1}, \ldots, x_{2^{\prime}}\right)\right],
$$

then for $t>2$ the two-generator group $G_{2}$ (constructed for this $V$ and for a countable group $G$ of length $l$ ) cannot be of length $l+2$ because, if this were the case, the verbal subgroup $V\left(G_{2}\right)$ of $G_{2}$ would be of length at most $l+2-t$. But such a group cannot contain a group of length $l$.

Nevertheless a shorter version of our construction of Section 2 enables us to exhibit another embedding, with the same properties apart from 'verbality'. 
THEOREM 2. For each countable group $G$, there exists a two-generator group $G_{2}$ in the variety $\operatorname{var}(G) \mathfrak{A}^{2}$ with a subnormal subgroup $\tilde{G}$ isomorphic to $G$. If $G$ is a fully ordered group the group $G_{2}$ can be fully ordered in such a way that the reduction of its order on $\tilde{G}$ makes the latter order isomorphic to $G$.

In particular, if the group $G$ is soluble of length $l$, the two-generator group $G_{2}$ is soluble of length $l+2$ but not $l+1$ in general.

If moreover the group $G$ (soluble of length $l$ ) is torsion free, the group $G_{2}$ (soluble of length $l+2)$ can be chosen to also be torsion free.

As we mentioned in the introduction this theorem strengthens results of [20], of [22], and of [1].

The proof of Theorem 2 is based on the constructions of Lemma 4 and Lemma 5. To avoid repetition we only sketch it. We note that this construction is more economical than that of Section 3 in [20], for at each step in each wreath product we take a smaller part of the base group than in [20] (and not all elements of the base group with well-ordered supports).

PROOF OF THEOREM 2. First we embed the given fully ordered group $G$ subnormally into a group $D$ so that $G \leq D^{\prime}$, and continue the full order of $G$ on $D$ as in Lemma 4. Next we embed the group $D$ into the wreath product $D \mathrm{Wr} Z$ (where $Z=\langle z\rangle$ is an infinite cycle) and choose an element $\omega \in D^{Z}$ as in the previous section. Then the two-generator group

$$
G_{2}=\left\langle\omega, z^{-1}\right\rangle
$$

contains the derived group of the first copy of $D$ in $D^{Z}$. Thus, as in Lemma $5, G$ is subnormal in $G_{2}$ of defect 4 , and the full order $D$ can be continued to a full order on $G_{2}$. Clearly, $G_{2} \in \operatorname{var}(G) \mathfrak{A} \mathfrak{A}=\operatorname{var}(G) \mathfrak{A}^{2}$.

Let us turn to another problem. Lemma 2 and Lemma 3 establish verbal, subnormal embedding of an arbitrary infinite group into a group of the same cardinality. This embedding is of defect 2 , and it is very natural to ask whether or not this defect can be reduced to 1 , that is, whether or not this embedding can be normal.

Normal verbal embeddings of groups are of independent interest. The problem is formulated and solved for finite $p$-groups by Heineken in [5]. For the general criterion of normal verbal embeddability we refer to our Main Theorem in [6]. Here we give a result on normal verbal embeddings of ordered groups which strengthens the statement $\mathbf{A}$ of our Theorem 1 in [15].

THEOREM 3. For an arbitrary fully ordered group $G$ and arbitrary non-trivial word set $V$, there exists a fully ordered group $H$ of the same cardinality as $G$ with a 
subnormal subgroup $\tilde{G}$ of defect 2 such that $\tilde{G}$ lies in $V(H)$ and the reduction of the order of $H$ on the subgroup $\tilde{G}$ makes the latter order isomorphic to $G$.

If the group $G$ is soluble or torsion free, the group $H$ can be chosen to satisfy these properties.

If the group $G$ is finitely generated, the group $H$ can be chosen to be finitely generated as well.

The defect of embedding cannot in general be made smaller: for some fully ordered group a normal verbal embedding cannot be obtained.

PROOF. The embedding needed is already constructed in Lemma 2 and Lemma 3. So it suffices to take $H=G_{1}$.

Assume $G$ is generated by finitely many elements $g_{1}, g_{2}, \ldots, g_{t}$. Denote by $\tilde{g}_{i}$ $(i=1, \ldots, t)$ the image of $g_{i}$ in $\tilde{G}$. For each of these elements there exist words $w_{i, j} \in V\left(j=1, \ldots, s_{i}\right)$ and elements $h_{i, j}^{k} \in H\left(k=1, \ldots, l_{i, j}\right)$ such that:

$$
g_{i}=\left(w_{i, 1}\left(h_{i, 1}^{1}, \ldots, h_{i, 1}^{l_{i, 1}}\right)\right)^{\epsilon_{i, 1}} \cdots\left(w_{i, s_{i}}\left(h_{i, s_{i}}^{1}, \ldots, h_{i, s_{i}}^{l_{i, s_{i}}}\right)\right)^{\epsilon_{i, s_{i}}}
$$

( $i=1, \ldots, t ; \epsilon_{i, 1}, \ldots, \epsilon_{i, s_{i}}= \pm 1$ ). So if the group $H$ is not finitely generated, it is enough to replace it by the finitely generated group

$$
\left\langle h_{i, j}^{k} \in H \mid i=1, \ldots, t ; j=1, \ldots, s_{i} ; k=1, \ldots, l_{i, j}\right\rangle,
$$

which contains the subgroup $\tilde{G}$.

Our criterion of normal verbal embeddability [6] enables us to construct an example of a group $G$ which for a certain word set $V$ cannot be normally embedded into a group $H$ such that $G \subseteq V(H)$, even without requiring $H$ to be fully ordered, torsion free, soluble or of the same cardinality as $G$ ! But since none of the examples in [6] is a torsion free fully ordered group-fully ordered groups must be torsion free-we give here a scheme of construction of fully ordered and 'not $n V$-embeddable' (see [6]) groups.

Every absolutely free group $F_{n}$ can be fully ordered [19]. Let $\mathfrak{V}$ be a non-abelian variety such that the group of automorphisms

$$
A=\operatorname{Aut}\left(F_{n}(\mathfrak{V})\right)
$$

of the $\mathfrak{V}$-free group of rank $n$ has a non-trivial identity $w \equiv 1$. It is easy to find examples of such varieties: it suffices to take a locally finite variety $\mathfrak{V}$. Then the group of automorphisms $A$ is finite and we can take $w=x^{k}$, where $k=\exp A$. Then

$$
\operatorname{Inn}\left(F_{n}\right) \mathbb{E}\left(\operatorname{Aut}\left(F_{n}\right)\right)^{k} \text {, }
$$

and this condition guarantees, by the Main Theorem in [6], that the fully ordered group $F_{n}$ is not normally embeddable into a group $H$ such that its image lies in $H^{k}=\left\langle x^{k} \mid x \in H\right\rangle$. 
In fact we could easily find many other examples of word sets $V$ with this property. And we could find other fully ordered groups which do not posses normal verbal embeddings; in particular such groups can even be nilpotent. Let $G=F_{n}\left(\mathfrak{N}_{c}\right)$ and $\mathfrak{V}$ be a subvariety of $\mathfrak{N}_{c}$ such that Aut $\left(F_{n}(\mathfrak{V})\right)$ satisfies a non-trivial identity $w=1$. It suffices to take as $\mathfrak{V}$ the variety defined by the identities

$$
\left[x_{1}, \ldots, x_{c+1}\right]=1 \text { and } x^{l}=1
$$

for some $l>2$. Then $F_{n}(\mathfrak{V})$ is finite and we can take $w=x^{k}$, where $k=$ exp Aut $\left(F_{n}(\mathfrak{V}) \text { ). Again } \operatorname{Inn}(G) \nsubseteq \text { (Aut }(G)\right)^{k}$ and so $G$ is not normally embeddable into a group $H$ such that its image lies in $H^{k}$.

The last theorem and this consideration gives rise to following problem.

PROBLEM. For the given non-trivial word set $V$ and fully ordered group $G$, find a criterion under which $G$ can be normally embedded into an appropriate fully ordered group $H$ with a subgroup $\tilde{G}$ such that $\tilde{G}$ lies in $V(H)$ and the reduction of the order of $H$ on $\tilde{G}$ makes the latter order isomorphic to $G$.

In this direction we restrict ourselves to Theorem 3 because this problem will be considered elsewhere [18].

\section{References}

[1] R. Dark, 'On subnormal embedding theorems of groups', J. London Math. Soc. 43 (1968), 387-390.

[2] L. Fuchs, Partially ordered algebraic systems (Pergamon Press, Oxford, London, 1963).

[3] P. Hall, 'The frattini subgroups of finitely generated groups', Proc. London Math. Soc. (3) 11 (1961), 327-352.

[4] __ 'On the embedding of a group into a join of given groups', J. Austral. Math.Soc. 17 (1974), 434-495.

[5] H. Heineken, 'Normal embeddings of $p$-groups into p-groups', Proc. Edinburgh Math. Soc. 35 (1992), 309-314.

[6] H. Heineken and V. H. Mikaelian, 'On normal verbal embeddings of groups', J. Math. Sci., New York 100 (2000), 1915-1924.

[7] G. Higman, B. Neumann and Hanna Neumann, 'Embedding theorems for groups', J. London Math. Soc. (3) 24 (1949), 247-254.

[8] I. Kaplanski, Infinite abelian groups (Univ. Michigan Publ., Ann Arbor, MI, 1954).

[9] M. I. Kargapolov and Ju. I. Merzlyakov, Fundamentals of the theory of groups, 3rd Edition, (Russian); English translation of the 2nd Edition by R. G. Burns (Springer, New York, 1979) (Nauka, Moscow, 1982).

[10] L. G. Kovács and B. H. Neumann, 'An embedding theorem for some countable groups', Acta Sci. Math. (Szeged) 26 (1965), 139-142.

[11] F. W. Levi, 'Ordered groups', Proc. Indian Acad. Sci. 16 (1942), 256-263.

[12] _-, 'Contributions to the theory of ordered groups', Proc. Indian Acad. Sci. 17 (1943), 199-201. 
[13] F. Levin and G. Rosenberger, 'A class of SQ-universal groups', in: Group theory, Singapore, 1987 (de Gruyter, Berlin, 1989) pp. $409-415$.

[14] V. H. Mikaelian, 'Embeddings of countable groups into 2-generator groups with additional properties', in: Abstracts of the International Congress of Mathematicians, Berlin (1998) pp. 31.

[15] —-, 'Subnormal embedding theorems for groups', J. London Math. Soc. 62 (2000), 398-406.

[16] —. 'On varieties of groups generated by wreath products of abelian groups', in: Abelian groups, rings and modules (Perth, 2000), Contemp. Math. 273 (Amer. Math. Soc., Providence, RI, 2001) pp. 223-238.

[17] — 'On embeddings of countable generalized soluble groups in two-generated groups', $J$. Algebra 250 (2002), 1-17.

[18] - 'Über die normalen Einbettungen der geordneten Gruppen', (in preparation) (German).

[19] B. H. Neumann, 'On ordered groups', Amer. J. Math. 71 (1949), 1-18.

[20] — 'Embedding theorems for ordered groups', J. London Math. Soc. 35 (1960), 503-512.

[21] —_, 'Embedding theorems for groups', Nieuw Arch. Wisk. (3) 16 (1968), 73-78.

[22] B. H. Neumann and Hanna Neumann, 'Embedding theorems for groups', J. London Math. Soc. 34 (1959), 465-479.

[23] Hanna Neumann, Varieties of groups (Springer, Berlin, 1968).

[24] P. M. Neumann, 'On the structure of standard wreath products of groups', Math. Z. 84 (1964), 343-373.

[25] A. Yu. Ol'shanskii, 'Efficient embeddings of countable groups', Vestnik Moskov. Univ. Ser. I Mat. Mekh. 105 (1989), 28-34 (Russian).

[26] —_, 'Embedding of countable periodic groups in simple 2-generator periodic groups', $U$ krain. Mat. Zh. 43 (1991), 980-986 (Russian); English translation: Ukrainian Math. J. 43 (1992), 914919.

[27] D. J. S. Robinson, A course in the theory of groups, 2nd Edition (Springer, New York, 1996).

[28] J. S. Wilson and P. A. Zalesskii, 'An embedding theorem for certain residually finite groups', Arch. Math. (Basel) 67 (1996), 177-182.

Department of Informatics and Computer Science

Yerevan State University

375025 Yerevan

Armenia

e-mail: mikaelian@e-math.ams.org 